\title{
State Tracking of MRAC Systems in the Presence of Controller Temporary Failure Based on a Switching Method
}

\author{
Caiyun $\mathrm{Wu}^{1,2}$ and Jun $\mathrm{Zhao}^{1}$ \\ ${ }^{1}$ State Key Laboratory of Synthetical Automation for Process Industries, Northeastern University, Shenyang 110819, China \\ ${ }^{2}$ School of Equipment Engineering, Shenyang Ligong University, Shenyang 110159, China \\ Correspondence should be addressed to Jun Zhao; zhaojun@mail.neu.edu.cn
}

Received 19 July 2013; Accepted 5 September 2013

Academic Editor: Bochao Zheng

Copyright (c) 2013 C. Wu and J. Zhao. This is an open access article distributed under the Creative Commons Attribution License, which permits unrestricted use, distribution, and reproduction in any medium, provided the original work is properly cited.

\begin{abstract}
The state tracking problem for a class of model reference adaptive control (MRAC) systems in the presence of controller temporary failures is studied. Due to the controller temporary failure, the considered system is viewed as an error switched system. The properties of Lyapunov function candidates without switching are described. Then the notion of global practical stability of switched systems is presented, and sufficient conditions for global practical stability of the error system under the restrictions of controller failure frequency and unavailability rate are provided. An example is presented to demonstrate the feasibility and effectiveness of the proposed method.
\end{abstract}

\section{Introduction}

There are often parameter, structural, and environment uncertainties in practical systems [1-4]. Model reference adaptive control (MRAC) has been used as an important control approach for such uncertain systems $[5,6]$. Closed-loop signal boundedness and asymptotic tracking can be ensured by a state feedback controller and adaptive laws in MRAC systems [7].

On the other hand, controller temporary failures are often encountered in real control systems due to various environment factors during operation. Some motivations of studying controller failures are summarized in [8]. The reasons can be roughly classified into two categories: passive and positive ones. A typical passive reason is that the signals are not transmitted perfectly or the controller itself is not available for some reasons. For instance, the packet dropout phenomenon in networked control systems leads to controller failure, which is inevitable because of unreliable transmission paths. In contrast to passive reasons, a typical positive reason is that the controller is purposefully suspended from time to time for an economic or system life consideration [9]. Apparently, controller failures may lead to severe performance deterioration of systems. Especially, for adaptive control systems, the controller failure may cause the tracking error divergence due to the uncertainties of systems. Therefore, it is both theoretically and practically important to develop some new techniques to deal with the case of controller temporary failure of adaptive systems.

Recently, there are rapidly growing interests in switched systems and switching control in the control community [1013]. In the study of stability of switched systems, one of the effective tools is the average dwell time approach [14-16]. Based on this approach, exponential stability is guaranteed if the unavailability rate of the controller is smaller than a specified constant and the average time interval between controller failures is large enough $[9,17,18]$. In [8], this result was further extended to symmetric linear time-invariant system. The concept of controller failure frequency was first introduced in [9], and the cases of controller temporary failure for a class of time-varying delay systems were analyzed in [19]. Interestingly, a nonswitched MRAC system in the presence of controller temporary failure can be viewed as a switched system with a switching signal depending on the time interval between controller failures. Thus, theories and methods of switched systems may be applicable to the study of the state tracking problem for nonswitched MRAC systems with controller temporary failure. However, this issue has been rarely explored so far. 
In this paper, we study the state tracking problem for MRAC systems in the presence of controller temporary failure. As in [8], the controller temporary failure means that the controller itself is not available or the controller signals are not transmitted perfectly within a certain time interval. Furthermore, we assume that the parameter estimation is "frozen" in the instant of the controller temporary failure until the controller works normally. There are two main issues to be addressed in this paper. One is to describe a tradition MRAC system in the presence of controller temporary failure as an error switched system with two subsystems: the normal error subsystem which stands for the case without controller failure and the unstable error subsystem which describes the case of controller failure. The other issue is the stability analysis for the error switched system. To address the second issue, we analyze the properties of Lyapunov function candidates without switching and introduce the notions of global practical stability, failure frequency, and unavailability rate.

The results in this paper have three features. First of all, MRAC systems in the presence of controller temporary failure are first considered. Secondly, the state tracking problem is studied from a switched system point of view. Finally, the global practical stability criterion is given for the considered system under the condition of controller failure frequency and unavailability rate.

The organization of the paper is as follows. The state tracking problem in the presence of controller failure is formulated in Section 2. In Section 3, we present an error switched system. Section 4 gives three lemmas and the main result. An example is given to illustrate the effectiveness of the proposed method in Section 5. Finally, the conclusions are presented in Section 6.

The notation is standard. Consider the following:

$\lambda_{\text {max }}(A)\left(\lambda_{\min }(A)\right)$ : the largest (smallest) eigenvalue of matrix $A$;

$$
\begin{aligned}
& \|A\|=\sqrt{\lambda_{\max }\left(A A^{T}\right)}: \text { the norm of matrix } A ; \\
& \|x\|=\sqrt{\left(\sum_{i=1}^{n}\left|x_{i}\right|^{2}\right)} \text { : the norm of a vector } x= \\
& \left(x_{1}, x_{2}, \ldots, x_{n}\right)^{T} \in R^{n} ;
\end{aligned}
$$

$\operatorname{tr}[A]$ : the trace of a square matrix $A$.

\section{Problem Statement}

Consider a system

$$
\dot{x}(t)=\Theta f(x)+B u(t),
$$

where $B \in R^{n \times n}$ is input matrix, $x(t) \in R^{n}$ is the state, $u(t) \in R^{n}$ is the control input, $\Theta \in R^{n \times p}$ is an uncertain constant parameter matrix with a bounded $\delta_{\Theta}$, that is, $\|\Theta\| \leq$ $\delta_{\Theta}$, and $f(x) \in R^{p}$ is a vector which can be described as $f(x)=F x+g(x)$, where $F \in R^{p \times n}$ is a constant parameter matrix and $\|g(x)\| \leq M$ for some $M \geq 0$.

The classical state tracking problem is to design a controller such that the state $x(t)$ of the system (1) tracks a given reference state $x_{m}(t)$ generated from the reference model system

$$
\dot{x}_{m}(t)=A_{m} x_{m}(t)+B_{m} r(t),
$$

where $A_{m} \in R^{n \times n}$ is a constant Hurwitz matrix, $B_{m} \in R^{n \times n}$ is a constant input matrix, and $r(t) \in R^{n}$ is a bounded and piecewise continuous reference input.

Suppose that there exist matrices $R, T$, and $W^{*}$ such that the following matching equations are satisfied:

$$
A_{m}=B R, \quad B_{m}=B T, \quad B W^{*}=\Theta,
$$

where $W^{*}$ is an unknown matrix due to uncertain constant parameter matrix $\Theta$.

Define the tracking error $e(t)=x_{m}(t)-x(t)$. To solve the state tracking problem, we use the controller structure [20]

$$
u(t)=\psi \omega
$$

where $\psi=\left[\begin{array}{lll}R & \widehat{W}(t) & T\end{array}\right]$ and $\omega=\left[\begin{array}{c}x^{T} \\ -f^{T}(x) \\ r^{T}\end{array}\right], \widehat{W}(t)$ is the estimate of unknown matrix $W^{*}$, and $\|\widehat{W}(t)\| \leq \delta_{\widehat{W}}$.

Apply a parameter projection adaptive law

$$
\dot{\widehat{W}}(t)=-\Gamma B^{T} P_{1} e(t) f^{T}(x)+F_{s},
$$

where $\Gamma=\operatorname{diag}\left\{\Gamma_{1}, \Gamma_{2}, \ldots, \Gamma_{i}, \ldots, \Gamma_{M}\right\}$ with positive constants $\Gamma_{i}, P_{1}$ is a symmetric positive definite matrix satisfying $A_{m}^{T} P_{1}+$ $P_{1} A_{m}<0$, and $F_{s}$ is a vector satisfying

$$
F_{s}= \begin{cases}0, & \text { if }\|\widehat{W}(t)\| \leq \delta_{\widehat{W}}, \\ \Gamma B^{T} P_{1} e(t) f^{T}(t), & \text { if }\|\widehat{W}(t)\|>\delta_{\widehat{W}} .\end{cases}
$$

Then, the closed-loop system is

$$
\dot{x}(t)=A_{m} x(t)+B_{m} r(t)+B \widetilde{W}(t) f(x),
$$

where $\widetilde{W}(t)=W^{*}-\widehat{W}(t)$.

From $[5,20,21], x(t)$ converges asymptotically to $x_{m}(t)$ under the controller (4) and the adaptive law (5), that is, $\lim _{t \rightarrow \infty} e(t)=0$.

We now consider the case of controller temporary failure depicted in Figure 1. Controller failures occur when the controller (a) itself is not available or when the signals are not transmitted perfectly on the route (b). Suppose that the time interval of the controller failures is not more than a specified constant $T_{s}$, which means the designed controller can be recovered within a finite time interval [19]. Also, the failed controller implies the complete breakdown of the controller $(u(t)=0)$ in its failure time interval [8]. Hence, the system (1) with the controller temporary failure is dominated by the following piecewise differential equations:

$$
\dot{x}(t)= \begin{cases}\Theta f(t)+B u(t), & \text { when the controller works, } \\ \Theta f(t), & \text { when the controller fails. }\end{cases}
$$

We introduce the following definitions which will play key roles in deriving our main results. 


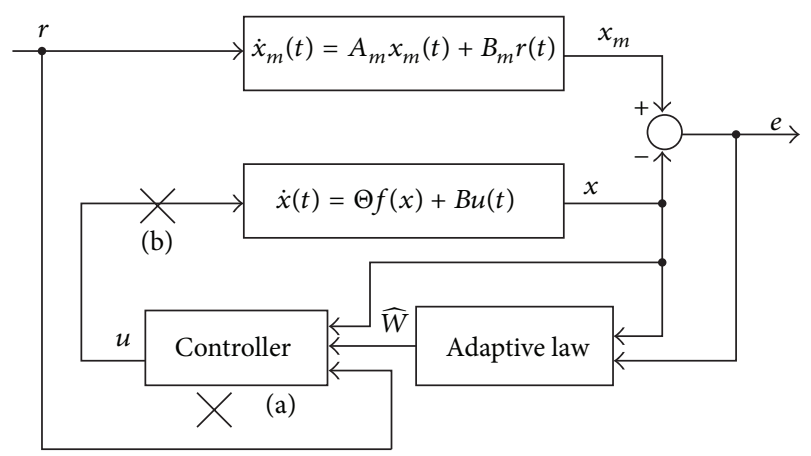

FIGURE 1: Controller failures occur in MRAC systems.

Definition 1 (see [8]). For any $T_{2}>T_{1}$, denote $T^{+}\left(T_{1}, T_{2}\right)$ as the total time interval of controller failure during $\left(T_{1}, T_{2}\right)$, and call the ratio $T^{+}\left(T_{1}, T_{2}\right) /\left(T_{2}-T_{1}\right)$ the unavailability rate of the controller in the system.

Definition 2 (see [9]). For any $T_{2}>T_{1} \geq 0$, let $N_{f}\left(T_{1}, T_{2}\right)$ denote the number of control failure in the time interval $\left(T_{1}, T_{2}\right) . F_{f}\left(T_{1}, T_{2}\right)=N_{f}\left(T_{1}, T_{2}\right) /\left(T_{2}-T_{1}\right)$ is referred to as the controller failure frequency in the time interval $\left(T_{1}, T_{2}\right)$.

In this paper, our objective is to develop conditions under which $x(t)$ tracks $x_{m}(t)$ subject to controller temporary failure.

\section{Error Switched System}

From the closed-loop system (7), we have a normal error system

$$
\dot{e}(t)=A_{m} e(t)-B \widetilde{W}(t) f(x) .
$$

When controller fails, we obtain an unstable error system

$$
\dot{e}(t)=A_{m} e(t)+A_{m} x(t)+B_{m} r-\Theta f(x) .
$$

In this condition, we choose the adaptive law

$$
\dot{\widehat{W}}(t)=0 \text {. }
$$

Remark 3. When controller fails, because the adaptive parameter $\widehat{W}(t)$ has no influence on the tracking error $e(t)$, it is proper that the parameter estimation $\widehat{W}(t)$ is "frozen" in the instants of the controller temporary failure until the controller works normally.

Based on (5), (9), (10), and (11), e(t) is governed by the following error switched system:

$$
\dot{e}(t)=A_{m} e(t)+\Phi_{\sigma}(t)
$$

where $\sigma(t):[0,+\infty) \rightarrow M=\{1,2\}, \Phi_{1}=-B \widetilde{W}(t) f(x)$, and $\Phi_{2}=A_{m} x(t) B_{m} r-\Theta f(x)$.

Meanwhile, we have a switching adaptive law of the following form:

$$
\dot{\widehat{W}}(t)=\left(-\Gamma B^{T} P_{1} e(t) f^{T}(x)+F_{s}\right) \Psi_{\sigma}
$$

where $\Psi_{1}=I_{p \times p}$ and $\Psi_{2}=0_{p \times p}$.
When $\sigma=1$, the normal error subsystem is active, which corresponds to the case of no controller failure; when $\sigma=2$, the unstable error subsystem is active, which denotes that the controller fails.

Therefore, the problem of state tracking in the presence of controller temporary failure can be handled by means of analyzing the stability of the error switched system (12) with the switching adaptive law (13).

To analyze the stability of the error switched system (12), we introduce the following definition.

Definition 4 (see [22]). Consider system (12). Given a constant $r^{*}>0$, the system (12) is said to be globally practically stable with respect to $r^{*}$ if there exist a switching law $\sigma(t)$ and a constant $T=T\left(e\left(t_{0}\right), r^{*}\right) \geq 0$ which depends on $e\left(t_{0}\right)$ and $r^{*}$ such that $e\left(t ; t_{0}, e\left(t_{0}\right)\right) \in S\left(r^{*}\right) \triangleq\left\{e \mid\|e\| \leq r^{*}\right\}$ for $t \geq t_{0}+T$.

Remark 5. Unlike the $\varepsilon$-practical stability concept [23], the initial error in Definition 4 is not required to be bounded. If the initial error is constrained by $e\left(t_{0}\right) \in B\left(r^{*}\right)$, then the global practical stability, given by Definition 4 , degenerates into $\mathcal{\varepsilon}$-practical stability [22]. Global practical stability stated here expresses a global version of the existing practical stability concept. Obviously, Definition 4 covers the $\varepsilon$-practical stability as a special case.

\section{Main Result}

In this section, firstly, we give three lemmas to analyze the properties of Lyapunov function candidates without switching. Secondly, we present a theorem to give some conditions under which the error switched system (12) is globally practically stable. Let

$$
z(t)=\left[e^{T}(t), \widetilde{w}_{1}^{T}(t), \ldots \widetilde{w}_{n}^{T}(t), \ldots \widetilde{w}_{p}^{T}(t)\right]^{T} \in R^{n+n \times p},
$$

where $\widetilde{w}_{i}(t) \in R^{n}$ is the $i$ th column of $\widetilde{W}(t), i=1,2, \ldots, p$; that is,

$$
\widetilde{W}(t)=\left[\widetilde{w}_{1}(t), \ldots, \widetilde{w}_{p}(t)\right] \in R^{n \times p} .
$$

According to [21], we have

$$
\operatorname{tr}\left[\widetilde{W}^{T} \Gamma^{-1} F_{s}\right] \leq 0
$$

Note that the parameter estimates $\widehat{W}$ are bounded; thus there exists a constant $l>0$ defined as

$$
l=\max \left(\operatorname{tr}\left[\widetilde{W}^{T} \Gamma^{-1} \widetilde{W}\right]\right) .
$$

Consider the situation of the system (1) without controller failure. A Lyapunov functional candidate of the normal error subsystem of (12) is chosen as

$$
V_{1}(z(t))=e^{T}(t) P_{1} e(t)+\operatorname{tr}\left[\widetilde{W}^{T}(t) \Gamma^{-1} \widetilde{W}(t)\right] .
$$

Differentiating $V_{1}$ along the trajectory of the normal error subsystem of (12) and the adaptive law (13) gives

$$
\dot{V}_{1}(z(t))=e^{T}(t)\left(A_{m}^{T} P_{1}+P_{1} A_{m}\right) e(t)+2 \operatorname{tr}\left[\widetilde{W}^{T} \Gamma^{-1} F_{s}\right] .
$$


The following lemma gives the estimate of the convergence rate of $V_{1}$ along the trajectory of the normal error subsystem of (12).

Lemma 6. Consider the normal error subsystem of (12). For any given $r_{1}>\sqrt{l / \lambda_{\min }\left(P_{1}\right)}$, denote $S\left(r_{1}\right)=\left\{e \mid\|e\|<r_{1}\right\}$. If $e(t) \in R^{n} / S\left(r_{1}\right)$, then the inequality

$$
V(z(t)) \leq \exp \left(-2 \lambda_{1}\left(t-t_{0}\right)\right) V_{1}\left(z\left(t_{0}\right)\right)
$$

holds for any $\lambda_{1}$ satisfying $0<\lambda_{1} \leq\left(\overline{\lambda_{1}} / 2\right)\left(1-\left(l / r_{1}^{2} \lambda_{\min }\left(P_{1}\right)\right)\right)$.

Proof. From (17) and (18), it is easy to get

$$
e^{T}(t) P_{1} e(t) \leq V_{1}(z(t)) \leq e^{T}(t) P_{1} e(t)+l .
$$

It is obvious that

$$
\lambda_{\text {min }}\left(P_{1}\right)\|e(t)\|^{2} \leq V_{1}(z(t)) \leq \lambda_{\max }\left(P_{1}\right)\|e(t)\|^{2}+l .
$$

From (16) and (19), it holds that

$$
\dot{V}_{1}(z(t)) \leq e^{T}\left(A_{m}^{T} P_{1}+P_{1} A_{m}\right) e<0 .
$$

Since $A_{m}$ is Hurwitz matrix, there exists a scalar $\overline{\lambda_{1}}>0$ such that $A_{m}^{T} P_{1}+P_{1} A_{m}+\overline{\lambda_{1}} P_{1}<0$.

With the help of (21)- (23), we have

$$
\begin{aligned}
& \dot{V}_{1}(z(t)) \\
& \quad \leq-\overline{\lambda_{1}} V_{1}(z(t))+\overline{\lambda_{1}} l \\
& \quad=-2 \lambda_{1} V_{1}(z(t))-\left(\overline{\lambda_{1}}-2 \lambda_{1}\right) V_{1}(z(t))+\overline{\lambda_{1}} l \\
& \quad \leq-2 \lambda_{1} V_{1}(z(t))-\lambda_{\text {min }}\left(P_{1}\right)\left(\overline{\lambda_{1}}-2 \lambda_{1}\right)\|e(t)\|^{2}+\overline{\lambda_{1}} l .
\end{aligned}
$$

Given that $r_{1}>\sqrt{l / \lambda_{\text {min }}\left(P_{1}\right)}$, when $e(t) \in R^{n} / S\left(r_{1}\right)$, applying (24) leads to $\dot{V}_{1}(z(t)) \leq-2 \lambda_{1} V_{1}(z(t))$ that is, $V(z(t)) \leq$ $\exp \left(-2 \lambda_{1}\left(t-t_{0}\right)\right) V_{1}\left(z\left(t_{0}\right)\right)$; for any $\lambda_{1}$ satisfying $0<\lambda_{1} \leq$ $\left(\overline{\lambda_{1}} / 2\right)\left(1-\left(l / r_{1}^{2} \lambda_{\min }\left(P_{1}\right)\right)\right)$.

This completes the proof.

When the controller fails, for the unstable error subsystem of (12), we choose another Lyapunov functional candidate of the following form:

$$
V_{2}(z(t))=e^{T}(t) P_{2} e(t)+\operatorname{tr}\left[\widetilde{W}^{T}(t) \Gamma^{-1} \widetilde{W}(t)\right],
$$

where $P_{2}$ is a positive definite matrix.

Differentiating $V_{2}$ along the trajectory of the normal error subsystem of (12) and the adaptive law (13) gives

$$
\dot{V}_{2}(z(t))=\dot{e}(t) P_{2} e(t)+e(t) P_{2} \dot{e}(t) .
$$

Then, in the following lemma, we estimate the divergence rate of $V_{2}$ along the trajectory of the unstable error subsystem of (12).
Lemma 7. Consider the unstable error subsystem of (12). For any given $r_{2}>0$, denote $S\left(r_{2}\right)=\left\{e \mid\|e\|<r_{2}\right\}$. If $e(t) \in$ $R^{n} / S\left(r_{2}\right)$, then the inequality

$$
V(z(t)) \leq \exp \left(2 \lambda_{2}\left(t-t_{0}\right)\right) V_{2}\left(z\left(t_{0}\right)\right)
$$

holds for any $\lambda_{2}$ satisfying $0<\lambda_{2} \leq\left(1 / 2 \lambda_{\text {min }}\left(P_{2}\right)\right)\left(\left(\beta_{2} / r_{2}\right)+\right.$ $\beta_{1}$ ).

Proof. From (17), we have

$$
e^{T}(t) P_{2} e(t) \leq V_{2}(z(t)) \leq e^{T}(t) P_{2} e(t)+l .
$$

It is obvious that

$$
\lambda_{\text {min }}\left(P_{2}\right)\|e(t)\|^{2} \leq V_{2}(z(t)) \leq \lambda_{\max }\left(P_{2}\right)\|e(t)\|^{2}+l .
$$

From (10) and (26), we have

$$
\begin{aligned}
\dot{V}_{2}(z(t))= & e^{T}(t)\left(A_{m}^{T} P_{2}+P_{2} A_{m}\right) e(t) \\
& +2 e^{T}(t) P_{2}\left(A_{m} x_{m}+B_{m} r(t)-\Theta f(x)\right) \\
= & e^{T}(t)\left(A_{m}^{T} P_{2}-P_{2} A_{m}+2 \Theta F\right) e(t) \\
& +2 e^{T}(t) P_{2}\left(\left(A_{m}-\Theta F\right) x_{m}+B_{m} r(t)-\Theta g(x)\right) \\
= & e^{T}(t) P_{3} e(t)+e^{T}(t) \xi_{1}-e^{T}(t) P_{2} \Theta g(x),
\end{aligned}
$$

where $P_{3} \triangleq A_{m}^{T} P_{2}-P_{2} A_{m}+2 \Theta F$ and $\xi_{1} \triangleq 2 P_{2}\left(A_{m}-\Theta F\right) x_{m}+$ $B_{m} r(t)$.

Since $\Theta$ and $r(t)$ are bounded, we have

$$
\begin{aligned}
\dot{V}_{2}(z(t)) \leq & \|e(t)\|^{2}\left\|P_{3}\right\|+\|e(t)\|\left\|\xi_{1}\right\| \\
& +\|e(t)\|\left\|P_{2}\right\|\|\Theta\|\|g(x)\| \\
\leq & \|e(t)\|^{2}\left\|P_{3}\right\|+\|e(t)\|\left\|\xi_{1}\right\| \\
& +M\|e(t)\|\left\|P_{2}\right\|\|\Theta\| \\
\leq & \|e(t)\|^{2}\left\|P_{3}\right\|+\|e(t)\|\left(\left\|\xi_{1}\right\|+M\left\|P_{2}\right\|\|\Theta\|\right) .
\end{aligned}
$$

Then, it holds that

$$
\dot{V}_{2}(z(t)) \leq \beta_{1}\|e(t)\|^{2}+\beta_{2}\|e(t)\|,
$$

where $\beta_{1}=\left\|P_{3}\right\|$ and $\beta_{2}=\left\|\xi_{1}\right\|+M\left\|P_{2}\right\|\|\Theta\|$.

Therefore, for any $r_{2}>0$, when $e(t) \in R^{n} / S\left(r_{2}\right)$, then the inequality $\dot{V}_{2}(z(t)) \leq 2 \lambda_{2} V_{2}(z(t))$, that is, $V(z(t)) \leq$ $\exp \left(2 \lambda_{2}\left(t-t_{0}\right)\right) V_{2}\left(z\left(t_{0}\right)\right)$, holds for any $\lambda_{2}$ satisfying $0<\lambda_{2} \leq$ $\left(1 / 2 \lambda_{\min }\left(P_{2}\right)\right)\left(\left(\beta_{2} / r_{2}\right)+\beta_{1}\right)$.

This completes the proof.

Based on Lemmas 6 and 7, we have the following lemma.

Lemma 8. Consider the subsystems of (12). For any $r_{3}>0$, if $e(t) \in R^{n} / S\left(r_{3}\right)$, then the inequality

$$
\eta_{1}\|e(t)\|^{2} \leq V_{i}(z(t)) \leq \eta_{3}\|e(t)\|^{2},
$$

holds for $\eta_{1}=\min \left\{\lambda_{\min }\left(P_{1}\right), \lambda_{\min }\left(P_{2}\right)\right\}, \eta_{2}=\max \left\{\lambda_{\max }\left(P_{1}\right)\right.$, $\left.\lambda_{\max }\left(P_{2}\right)\right\}$, and $\eta_{3}=\eta_{2}+\left(l / r_{3}^{2}\right)$. 
Proof. Form (22) and (29), we have

$$
e^{T}(t) P_{i} e(t) \leq V_{i}(z(t)) \leq e^{T}(t) P_{i} e(t)+l .
$$

Denote that $\eta_{1}=\min \left\{\lambda_{\min }\left(P_{1}\right), \lambda_{\min }\left(P_{2}\right)\right\}$ and $\eta_{2}=$ $\max \left\{\lambda_{\max }\left(P_{1}\right), \lambda_{\text {max }}\left(P_{2}\right)\right\}$. Then, we have

$$
\eta_{1}\|e(t)\|^{2} \leq V_{i}(z(t)) \leq \eta_{2}\|e(t)\|^{2}+l .
$$

For any $r_{3}>0$, when $e(t) \in R^{n} / S\left(r_{3}\right)$, we can find a constant $\eta_{3}=\eta_{2}+\left(l / r_{3}^{2}\right)$ such that $\eta_{1}\|e(t)\|^{2} \leq V_{i}(z(t)) \leq$ $\eta_{3}\|e(t)\|^{2}$.

This completes the proof.

Furthermore, according to Lemmas $6-8$, for any given $r_{0}^{\prime}=\max \left\{r_{1}, r_{2}, r_{3}\right\}$ and $t>t_{0}$, if $e(t) \in R^{n} / S\left(r_{0}^{\prime}\right)$, it is true that

$$
\begin{aligned}
& V(z(t)) \\
& \leq \begin{cases}\exp \left(-2 \lambda_{1}\left(t-t_{0}\right)\right) V_{1}\left(z\left(t_{0}\right)\right) & \text { when the controller } \\
\exp \left(2 \lambda_{2}\left(t-t_{0}\right)\right) V_{2}\left(z\left(t_{0}\right)\right) & \text { works, } \\
& \text { when the controller }\end{cases}
\end{aligned}
$$

For (18) and (25), it is obvious that there exists $\mu=\eta_{3} / \eta_{1}$ such that

$$
V_{i}(z(t)) \leq \mu V_{j}(z(t)) \quad(i, j=1,2) .
$$

Without loss of generality, for $k=0,1, \ldots$, we assume that the controller works during $\left[t_{2 k}, t_{2 k+1}\right)$, which means that the first subsystem is active on $\left[t_{2 k}, t_{2 k+1}\right)$, while the controller fails during $\left[t_{2 k+1}, t_{2 k+2}\right)$, which denotes that the second subsystem is active on $\left[t_{2 k+1}, t_{2 k+2}\right)$.

When $e\left(t_{2 k}\right) \in S\left(r_{1}\right)$, from $(22)$, we have $e\left(t_{2 k+1}\right) \in S\left(r_{1}^{\prime}\right)$ for any given $r_{1}^{\prime} \geq \sqrt{\left(r_{1}^{2} \lambda_{\max }\left(P_{1}\right)+l\right) / \lambda_{\min }\left(P_{1}\right)}$. It is obvious that (36) and (37) still hold for $r_{0} \geq \max \left\{r_{1}^{\prime}, r_{0}^{\prime}\right\}$.

Remark 9. Because of the uncertainties of the systems, the properties of the Lyapunov function candidates are restricted outside the ball with the radius $r_{0}$ as described as in Lemmas 6-8. paper.

Now, we are in the position to give the main result of this

Theorem 10. Consider the error switched system (12) with the adaptive control law (13). For $k=0,1, \ldots$, if the switching law $\sigma(t)$ satisfies the following two conditions:

\section{Condition 1}

$$
\frac{T^{+}\left(t_{2 k}, t\right)}{\left(t-t_{2 k}\right)} \leq \frac{\left(\lambda_{1}-\lambda^{*}\right)}{\left(\lambda_{1}+\lambda_{2}\right)}
$$

holds for some scalar $\lambda^{*} \in\left(0, \lambda_{1}\right)$,

\section{Condition 2}

$$
F_{f}\left(t_{2 k}, t\right) \leq F_{f}^{*}=\frac{\lambda}{\ln \mu}
$$

holds for some scalar $\lambda \in\left(0, \lambda^{*}\right)$, then, the error switched system (12) is globally practically stable.

Proof. For any given $r_{0} \geq \max \left\{r_{1}^{\prime}, r_{0}^{\prime}\right\}$, denote

$$
\begin{aligned}
r^{*}= & \exp \left[\|\Theta\|\|F\|\left(T_{s}\right)\right] \cdot r_{0} \\
& +\frac{(\kappa+M\|\Theta\|)\left[\exp \left(\|\Theta\|\|F\|\left(T_{s}\right)\right)-1\right]}{\|\Theta\|\|F\|},
\end{aligned}
$$

where $\kappa \geq\left\|\left(A_{m}-\Theta F\right) x_{m}(t)+B_{m} r(t)\right\|$ for some $\kappa>0$. When the initial error $e\left(t_{0}\right) \in R^{n}$, we will show that there exists a constant $T=T\left(e\left(t_{0}\right), r^{*}\right) \geq 0$, concerned with $e\left(t_{0}\right)$ and $r^{*}$ such that $e\left(t ; t_{0} e\left(t_{0}\right)\right) \in S\left(r^{*}\right)$ for $t \geq t_{0}+T$ under the switching law $\sigma(t)$ satisfying Conditions 1-2. To this end, we will prove the theorem in three cases.

(a) For $e\left(t_{2 k}\right) \in S\left(r_{0}\right)$, we will show that $e\left(t_{2 k+2}\right) \in S\left(r^{*}\right)$.

(b) For $e\left(t_{2 k}\right) \in R^{n} / S\left(r_{0}\right)$, we will prove that there exists $T_{2 k}=T_{2 k}\left(e\left(t_{2 k}\right), r_{0}\right) \geq 0$ such that $\left\|e\left(t_{2 k}+T_{2 k}\right)\right\|=r_{0}$ for $e(t) \in R^{n} / S\left(r_{0}\right)$ and $t \in\left[t_{2 k}, T_{2 k}\right)$.

(c) When the initial error $e\left(t_{0}\right) \in R^{n}$, we will show that there exists a constant $T=T\left(e\left(t_{0}\right), r^{*}\right) \geq 0$ such that $e\left(t ; t_{0}, e\left(t_{0}\right)\right) \in S\left(r^{*}\right)$ for $t \geq t_{0}+T$ under the switching law $\sigma(t)$ satisfying Conditions 1 and 2 .

We first prove (a). Consider $e\left(t_{2 k}\right) \in S\left(r_{0}\right)$. Because of the asymptotical stability of the normal subsystem of (12), it is obvious that $e\left(t_{2 k+1}\right) \in S\left(r_{0}\right)$. When $t \in\left[t_{2 k+1}, t_{2 k+2}\right)$, the second subsystem is active. From (10), we have

$$
\dot{e}(t)=\Theta F e(t)+\left(A_{m}-\Theta F\right) x_{m}+B_{m} r-\Theta g(x) ;
$$

then, the trajectory of the error switched system (12) satisfies

$$
\begin{aligned}
& e\left(t_{2 k+2}\right) \\
&=\exp \left[\Theta F\left(t_{2 k+2}-t_{2 k+1}\right)\right] e\left(t_{2 k+1}\right) \\
&+\int_{t_{2 k+1}}^{t_{2 k+2}}\left\{\exp \left[\Theta F\left(t_{2 k+2}-t\right)\right]\right. \\
&\left.\quad \times\left[\left(A_{m}-\Theta F\right) x_{m}(t)+B_{m} r(t)-\Theta g(x(t))\right]\right\} d t .
\end{aligned}
$$

With the help of $\left\|e^{A}\right\| \leq e^{\|A\|}$, it is obvious that

$$
\begin{aligned}
& \left\|e\left(t_{2 k+2}\right)\right\| \\
& \leq \exp \left[\|\Theta\|\|F\| \cdot\left(t_{2 k+2}-t_{2 k+1}\right)\right]\left\|e\left(t_{2 k+1}\right)\right\| \\
& +\int_{t_{2 k+1}}^{t_{2 k+2}}\left\{\exp \left[\|\Theta\|\|F\| \cdot\left(t_{2 k+2}-\tau\right)\right]\right. \\
& \times\left[\left\|\left(A_{m}-\Theta F\right) x_{m}(\tau)+B_{m} r(\tau)\right\|\right. \\
& +\|\Theta\|\|g(x(\tau))\|]\} d \tau
\end{aligned}
$$


$\leq \exp \left[\|\Theta\|\|F\|\left(t_{2 k+2}-t_{2 k+1}\right)\right]\left\|e\left(t_{2 k+1}\right)\right\|$

$+\int_{t_{2 k+1}}^{t_{2 k+2}}\left\{(\Lambda+M\|\Theta\|) \exp \left[\|\Theta\|\|F\|\left(t_{2 k+2}-\tau\right)\right]\right\} d \tau$

$=\exp \left[\|\Theta\|\|F\|\left(t_{2 k+2}-t_{2 k+1}\right)\right]\left\|e\left(t_{2 k+1}\right)\right\|$

$+\frac{(\Lambda+M\|\Theta\|)\left[\exp \left(\|\Theta\|\|F\|\left(t_{2 k+2}-t_{2 k+1}\right)\right)-1\right]}{\|\Theta\|\|F\|}$.

Because $T_{s}=\max _{k}\left\{t_{2 k+2}-t_{2 k+1}\right\}$, we have

$$
\begin{aligned}
\left\|e\left(t_{2 k+2}\right)\right\| \leq & \left\|e\left(t_{2 k+1}+T_{s}\right)\right\| \\
= & \exp \left[\|\Theta\|\|F\|\left(T_{s}\right)\right]\left\|e\left(t_{2 k+1}\right)\right\| \\
& +\frac{(\kappa+M\|\Theta\|)\left[\exp \left(\|\Theta\|\|F\|\left(T_{s}\right)\right)-1\right]}{\|\Theta\|\|F\|} .
\end{aligned}
$$
have

Note that $r_{0}$ is the maximum value of $\left\|e\left(t_{2 k+1}\right)\right\|$; thus we

$$
\begin{aligned}
\left\|e\left(t_{2 k+2}\right)\right\| \leq & r^{*} \\
= & \exp \left[\|\Theta\|\|F\|\left(T_{s}\right)\right] r_{0} \\
& +\frac{(\kappa+M\|\Theta\|)\left[\exp \left(\|\Theta\|\|F\|\left(T_{s}\right)\right)-1\right]}{\|\Theta\|\|F\|} .
\end{aligned}
$$

Because of $r^{*}>r_{0}$, we have $e\left(t_{2 k+2}\right) \in S\left(r^{*}\right)$ when $e\left(t_{2 k}\right) \in$ $S\left(r_{0}\right)$.

Then, we prove (b). For $e\left(t_{2 k}\right) \in R^{n} / S\left(r_{0}\right)$, obviously, we only need to consider the trajectory $e(t)$ being totally outside $S\left(r_{0}\right)$. We discuss two cases for $t \geq t_{2 k}$. One is $t \in\left[t_{2 j}, t_{2 j+1}\right)$, and the other is $t \in\left[t_{2 j+1}, t_{2 j+2}\right)$, where $j=k, k+1, \ldots$

Thus, from Lemmas 6 and 7 , it is true that

$V(z(\tau))$

$\leq \begin{cases}\exp \left(-2 \lambda_{1}\left(\tau-t_{2 k}\right)\right) V_{1}\left(z\left(t_{2 k}\right)\right), & \text { if } t_{2 k} \leq \tau<t_{2 k+1} \\ \exp \left(2 \lambda_{2}\left(\tau-t_{2 k+1}\right)\right) V_{2}\left(z\left(t_{2 k+1}\right)\right), & \text { if } t_{2 k+1} \leq \tau<t_{2 k+2} .\end{cases}$

If $t \in\left[t_{2 j}, t_{2 j+1}\right)$, according to (37) and (46), it holds that

$$
\begin{aligned}
V(z(t)) \leq & \exp \left(-2 \lambda_{1}\left(t-t_{2 j}\right)\right) V_{1}\left(z\left(t_{2 j}\right)\right) \\
\leq & \exp \left(-2 \lambda_{1}\left(t-t_{2 j}\right)\right) \mu V_{2}\left(z\left(t_{2 j}\right)\right) \\
\leq & \mu \exp \left(-2 \lambda_{1}\left(t-t_{2 j}\right)\right) \exp \left(2 \lambda_{2}\left(t_{2 j}-t_{2 j-1}\right)\right) \\
& \times V_{2}\left(z\left(t_{2 j-1}\right)\right) \\
\leq & \mu^{2} \exp \left(-2 \lambda_{1}\left(t-t_{2 j}\right)\right) \exp \left(2 \lambda_{2}\left(t_{2 j}-t_{2 j-1}\right)\right) \\
& \times V_{1}\left(z\left(t_{2 j-1}\right)\right)
\end{aligned}
$$

$$
\begin{gathered}
\leq \mu^{2} \exp \left(-2 \lambda_{1}\left(t-t_{2 j}\right)\right) \exp \left(2 \lambda_{2}\left(t_{2 j}-t_{2 j-1}\right)\right) \\
\times \exp \left(-2 \lambda_{1}\left(t_{2 j-1}-t_{2 j-2}\right)\right) V_{1}\left(z\left(t_{2 j-2}\right)\right) \\
\leq \cdots \leq \mu^{2(j-k)} \exp \left(-2 \lambda_{1}\left(t-t_{2 k}-T^{+}\left(t_{2 k}, t\right)\right)\right) \\
\quad \times \exp \left(2 \lambda_{2} T^{+}\left(t_{2 k}, t\right)\right) V_{1}\left(z\left(t_{2 k}\right)\right) .
\end{gathered}
$$

If $t \in\left[t_{2 j+1}, t_{2 j+2}\right)$, again from (37) and (46), we have

$$
\begin{aligned}
V(z(t)) \leq & \exp \left(2 \lambda_{2}\left(t-t_{2 j+1}\right)\right) V_{2}\left(z\left(t_{2 j+1}\right)\right) \\
\leq & \exp \left(2 \lambda_{2}\left(t-t_{2 j+1}\right)\right) \mu V_{1}\left(z\left(t_{2 j+1}\right)\right) \\
\leq & \mu \exp \left(2 \lambda_{2}\left(t-t_{2 j+1}\right)\right) \exp \left(-2 \lambda_{1}\left(t_{2 j+1}-t_{2 j}\right)\right) \\
& \times V_{1}\left(z\left(t_{2 j}\right)\right) \\
\leq & \mu^{2} \exp \left(2 \lambda_{2}\left(t-t_{2 j+1}\right)\right) \exp \left(-2 \lambda_{1}\left(t_{2 j+1}-t_{2 j}\right)\right) \\
& \times V_{2}\left(z\left(t_{2 j}\right)\right) \\
\leq & \mu^{2} \exp \left(2 \lambda_{2}\left(t-t_{2 j+1}\right)\right) \exp \left(-2 \lambda_{1}\left(t_{2 j+1}-t_{2 j}\right)\right) \\
& \times \exp \left(2 \lambda_{2}\left(t_{2 j}-t_{2 j-1}\right)\right) V_{2}\left(z\left(t_{2 j-1}\right)\right) \\
\leq & \cdots \leq \mu^{2(j-k)+1} \exp \left(-2 \lambda_{1}\left(t-t_{2 k}-T^{+}\left(t_{2 k}, t\right)\right)\right) \\
& \quad \times \exp \left(2 \lambda_{2} T^{+}\left(t_{2 k}, t\right)\right) V_{1}\left(z\left(t_{2 k}\right)\right) .
\end{aligned}
$$

By Definition 2, we know $N_{f}\left(t_{2 k}, t\right)=2(j-k)$ for $t \in$ $\left[t_{2 j}, t_{2 j+1}\right)$ and $N_{f}\left(t_{2 k}, t\right)=2(j-k)+1$ for $t \in\left[t_{2 j+1}, t_{2 j+2}\right)$. Thus, for any $t \in\left[t_{2 j+1}, t_{2(j+1)}\right)$, from (47) and (48), we can obtain

$$
\begin{aligned}
V(z(t)) \leq & \mu^{N_{f}\left(t_{2 k}, t\right)} \exp \left(-2 \lambda_{1}\left(t-t_{2 k}-T^{+}\left(t_{2 k}, t\right)\right)\right) \\
& \times \exp \left(2 \lambda_{2} T^{+}\left(t_{2 k}, t\right)\right) V_{1}\left(z\left(t_{2 k}\right)\right) .
\end{aligned}
$$

With the help of Lemma 8, it holds that

$$
\begin{aligned}
\|e(z(t))\| \leq & \sqrt{\frac{\eta_{2}}{\eta_{1}}} \exp \left(N_{f}\left(t_{2 k}, t\right) \ln \mu\right) \\
& \times \exp \left(-\lambda_{1}\left(t-t_{2 k}-T^{+}\left(t_{2 k}, t\right)\right)\right) \\
& \times \exp \left(\lambda_{2} T^{+}\left(t_{2 k}, t\right)\right)\left\|e\left(z\left(t_{2 k}\right)\right)\right\| .
\end{aligned}
$$

Applying Condition 1 gives

$$
\begin{aligned}
& \exp \left(-\lambda_{1}\left(t-t_{2 k}-T^{+}\left(t_{2 k}, t\right)\right)+\lambda_{2} T^{+}\left(t_{2 k}, t\right)\right) \\
& \quad \leq \exp \left(-\lambda^{*}\left(t-t_{2 k}\right)\right) .
\end{aligned}
$$

From Condition 2 and Definition 1, we have

$$
\exp \left(N_{f}\left(t_{2 k}, t\right) \ln \mu\right) \leq \exp \left(\lambda\left(t-t_{2 k}\right)\right)
$$


Using (49), (51), and (52) results in

$$
\begin{aligned}
& \left\|e\left(z\left(t ; t_{2 k}, e\left(t_{2 k}\right)\right)\right)\right\| \\
& \quad \leq \exp \left(-\left(\lambda^{*}-\lambda\right)\left(t-t_{2 k}\right)\right)\left\|e\left(z\left(t_{2 k}\right)\right)\right\| .
\end{aligned}
$$

Therefore, when $e\left(t_{2 k}\right) \in R^{n} / S\left(r_{0}\right)$, there exists $T_{2 k}=$ $T_{2 k}\left(e\left(t_{2 k}\right), r_{0}\right) \geq 0$ such that $\left\|e\left(t_{2 k}+T_{2 k}\right)\right\|=r_{0}$ under the switching law $\sigma(t)$ satisfying Conditions 1-2. Obviously, $\left\{T_{2 k}\right\}$ is a decreasing sequence, and thus $T_{0}=\max _{k}\left\{T_{2 k}\right\}$.

Finally, we prove (c). If $e\left(t_{0}\right) \in R^{n} / S\left(r^{*}\right)$, by applying (a), (b), and $r^{*}>r_{0}$, there exists a positive constant $T=$ $T\left(e\left(t_{0}\right), r^{*}\right)<T_{0}$ such that $e\left(t ; t_{0}, e\left(t_{0}\right)\right) \in S\left(r^{*}\right)$ for $t \geq t_{0}+T$. If $e\left(t_{0}\right) \in S\left(r^{*}\right)$, the result remains true with $T=0$.

This completes the proof.

Remark 11. When the initial error $e\left(t_{0}\right) \in S\left(r^{*}\right)$, the error switched system is $\mathcal{E}$-practical stability [23].

Remark 12. The error switched system (12) is globally practically stable if the controller fails only for a short time interval and with a low frequency of occurrence.

\section{Example}

In this section, we present an example to demonstrate the effectiveness of the proposed method in this paper.

Consider the system (1) with

$$
\begin{gathered}
\Theta=\left[\begin{array}{ll}
1 & 3 \\
1 & 2
\end{array}\right], \quad F=\left[\begin{array}{ll}
1 & 0 \\
0 & 1
\end{array}\right], \\
g(x)=\left[\begin{array}{l}
\sin x_{1} \\
\cos x_{2}
\end{array}\right], \quad B=\left[\begin{array}{ll}
1 & 0 \\
0 & 2
\end{array}\right] .
\end{gathered}
$$

The reference state $x_{m}$ is generated by the reference model (2) with $A_{m}=\left[\begin{array}{cc}-2 & 0 \\ 0 & -3\end{array}\right], B_{m}=\left[\begin{array}{l}1 \\ 1\end{array}\right]$, and the reference input is $r=1$.

Choose $R=\left[\begin{array}{cc}-2 & 0 \\ 0 & -1.5\end{array}\right], T=\left[\begin{array}{c}1 \\ 0.5\end{array}\right], P_{1}=P_{2}=\left[\begin{array}{cc}0.4449 & 0 \\ 0 & 0.2862\end{array}\right]$, and $\Gamma=\left[\begin{array}{cc}20 & 0 \\ 0 & 20\end{array}\right]$. We have $r^{*}=6.8147$ when $r_{0}=5$. Then, according to (38) and (39), we obtain $T^{+}\left(t_{2 k}, t\right) /\left(t-t_{2 k}\right) \leq$ 0.0302 and $F_{f}\left(t_{2 k}, t\right) \leq F_{f}^{*}=0.548$. The switching signal is chosen as

$$
\sigma(t)= \begin{cases}1, & t \in \Pi, \\ 2, & t \in \frac{[0,+\infty)}{\Pi},\end{cases}
$$

where $\Pi=[15 k, 15 k+1.95) \cup[15 k+2,15 k+4) \cup[15 k+$ $4.05,15 k+6.35) \cup[15 k+6.4,15 k+8.85) \cup[15 k+8.9,15 k+$ $11.55) \cup[15 k+11.6,15 k+14.55) \cup[15 k+14.6,15 k+15)$, $k=0,1,2, \ldots$, which is described in Figure 2 . It is easy to verify that $\sigma(t)$ satisfies Conditions 1-2 of Theorem 10.

When $x_{m}\left(t_{0}\right)=[0,0]^{T}$ and $x\left(t_{0}\right)=[5,6]^{T}$, the norm of the tracking error of (12) with and without controller failures is shown in Figures 3 and 4, respectively.

Simulations are carried out for $\left\|e\left(t_{0}\right)\right\|=7.81 \geq r_{0}$ and $\left\|e\left(t_{0}\right)\right\|=2.236<r^{*}$. The results are depicted in Figures 5 and 6 .

From Figures 5 and 6 , we can conclude that whether $\left\|e\left(t_{0}\right)\right\| \geq r_{0}$ or not, the states of the system (1) with controller

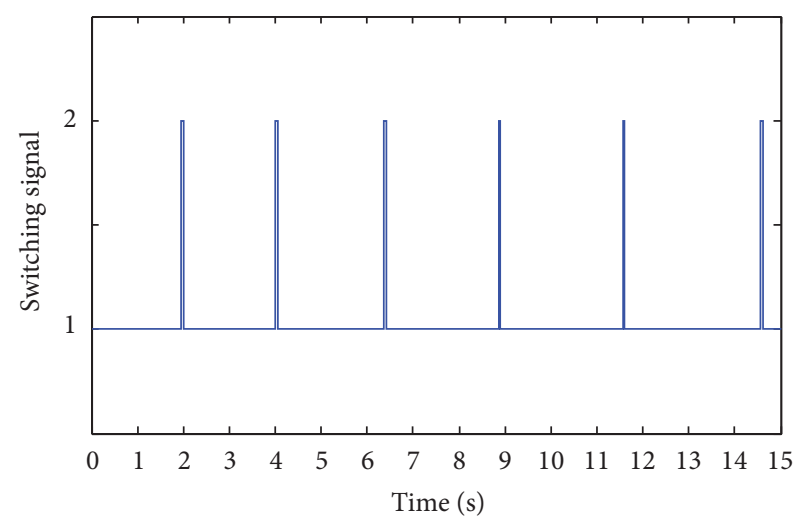

FIGURE 2: Switching signal.

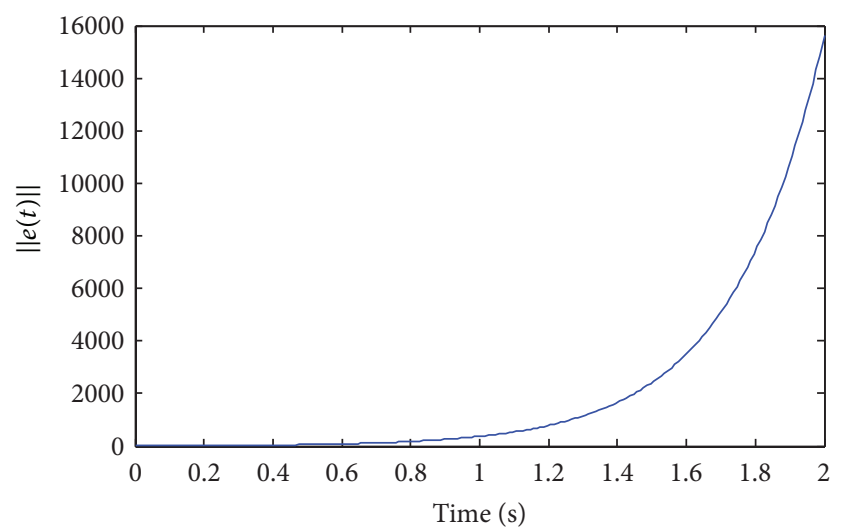

FIGURE 3: The norm of the tracking error of (12) when controller fails.

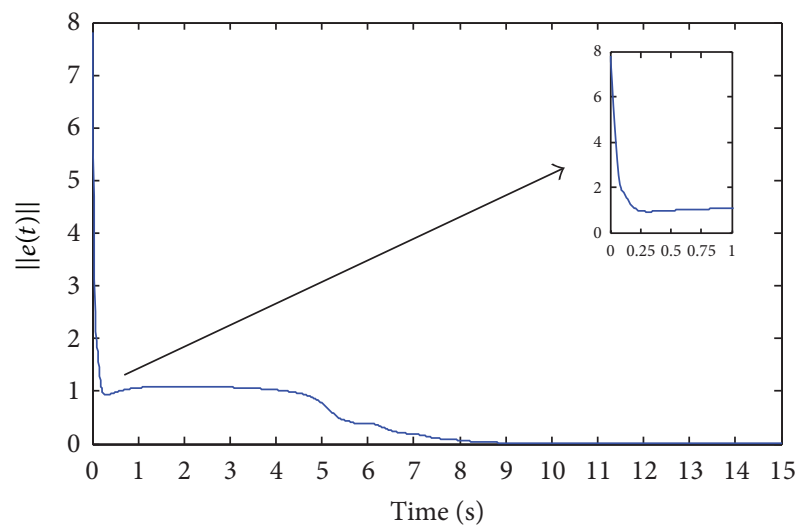

FIGURE 4: The norm of the tracking error of (12) without controller failure.

temporary failure track the reference signal $x_{m}(t)$ well under the switching signal $\sigma(t)$, and the tracking error $\|e(t)\|$ is small in the sense of $r^{*}$. Simulation illustrates the effectiveness of the proposed method.

\section{Conclusion}

This paper has considered the state tracking problem for a class of MRAC systems in the presence of controller 


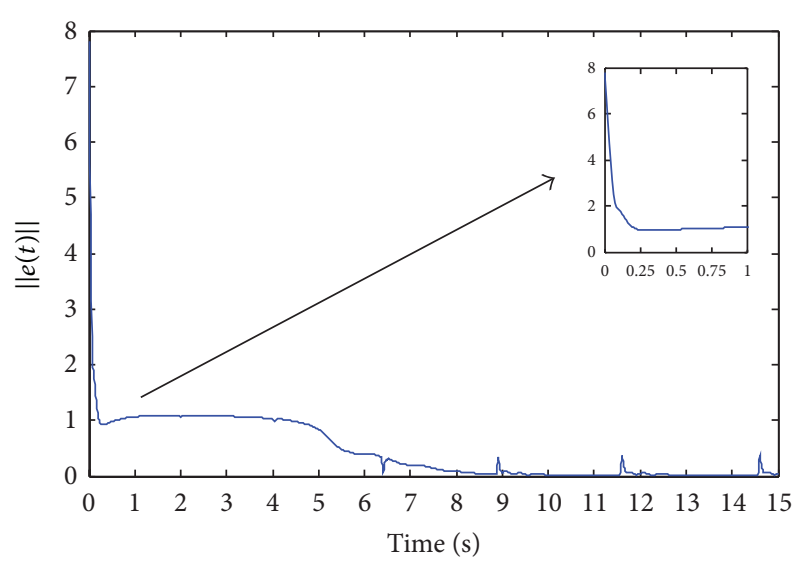

FIGURE 5: The norm of the tracking error of (12) under $\sigma(t)\left(\left\|e\left(t_{0}\right)\right\|=\right.$ $7.81)$.

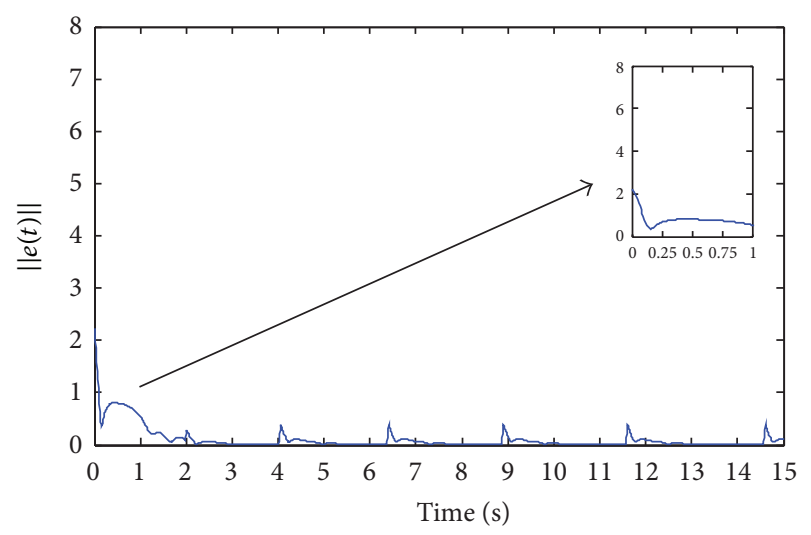

FIGURE 6: The norm of the tracking error of (12) under $\sigma(t)\left(\left\|e\left(t_{0}\right)\right\|=\right.$ 2.236).

temporary failure. A key point is to describe such a system as an error switched system. The properties of Lyapunov function candidates without switching have been given. Then, the global practical stability of the error switched system can be ensured by the proposed scheme, providing that the controller suffers from failures only for a relatively short time interval and with a low frequency of occurrence. It is an interesting topic to extend the results for the output tracking problem of adaptive systems.

\section{Conflict of Interests}

The authors declare that there is no conflict of interests regarding the publication of this paper.

\section{Acknowledgments}

This work was supported by the Chinese National Fundamental Research Program under Grant 2009CB320601 and National Natural Science Foundation of China under Grants 61233002 and 61174073.

\section{References}

[1] H. K. Khalil, "Adaptive output feedback control of nonlinear systems represented by input-output models," IEEE Transactions on Automatic Control, vol. 41, no. 2, pp. 177-188, 1996.

[2] S. Yin, X. Yang, and H. R. Karimi, "Data-driven adaptive observer for fault diagnosis," Mathematical Problems in Engineering, vol. 2012, Article ID 832836, 21 pages, 2012.

[3] Q. Sang and G. Tao, "Adaptive control of piecewise linear systems with state feedback for output tracking," Asian Journal of Control, vol. 15, no. 4, pp. 933-943, 2013.

[4] M. di Bernardo, U. Montanaro, and S. Santini, "Hybrid model reference adaptive control of piecewise affine systems," IEEE Transactions on Automatic Control, vol. 58, no. 2, pp. 304-316, 2013.

[5] P. A. Ioannou and J. Sun, Robust Adaptive Control, Prentice Hall, Englewood Cliffs, NJ, USA, 1996.

[6] B. M. Mirkin and P.-O. Gutman, "Adaptive coordinated decentralized control of state delayed systems with actuator failures," Asian Journal of Control, vol. 8, no. 4, pp. 441-448, 2006.

[7] G. Tao, Adaptive Control Design and Analysis, Adaptive and Learning Systems for Signal Processing, Communications, and Control, John Wiley \& Sons, Hoboken, NJ, USA, 2003.

[8] G. Zhai and H. Lin, "Controller failure time analysis for symmetric $H_{\infty}$ control systems," International Journal of Control, vol. 77, no. 6, pp. 598-605, 2004.

[9] X. M. Sun, G. P. Liu, D. Rees, and W. Wang, "Stability of systems with controller failure and time-varying delay," IEEE Transactions on Automatic Control, vol. 53, no. 10, pp. 2391-2396, 2008.

[10] D. Liberzon, Switching in Systems and Control, Systems \& Control: Foundations \& Applications, Birkhäuser, Boston, Mass, USA, 2003.

[11] J. Zhao and D. J. Hill, "Dissipativity theory for switched systems," IEEE Transactions on Automatic Control, vol. 53, no. 4, pp. 941-953, 2008.

[12] J. Li, Q. Su, and L. Sun, "Fault detection for nonlinear impulsive switched systems," Mathematical Problems in Engineering, vol. 2013, Article ID 815329, 12 pages, 2013.

[13] B. Niu and J. Zhao, "Barrier Lyapunov functions for the output tracking control of constrained nonlinear switched systems," Systems \& Control Letters, vol. 62, no. 10, pp. 963-971, 2013.

[14] J. P. Hespanha and A. S. Morse, "Stability of switched systems with average dwell-time," in Proceedings of the 38th IEEE Conference on Decision and Control, pp. 2655-2660, 1999.

[15] L. I. Allerhand and U. Shaked, "Robust stability and stabilization of linear switched systems with dwell time," IEEE Transactions on Automatic Control, vol. 56, no. 2, pp. 381-386, 2011.

[16] G. S. Zhai, B. Hu, K. Yasuda, and A. N. Michel, "Stability analysis of switched systems with stable and unstable subsystems: an average dwell time approach," International Journal of Systems Science, vol. 32, no. 8, pp. 1055-1061, 2001.

[17] G. S. Zhai, X. Chen, S. Takai, and K. Yasuda, "Controller failure time analysis for H-infinity control systems," in Proceedings of the 40th IEEE Conference on Decision and Control (CDC '01), pp. 1029-1030, December 2001.

[18] G. S. Zhai, B. Hu, K. Yasuda, and A. N. Michel, "Piecewise lyapunov functions for switched systems with average dwell time," Asian Journal of Control, vol. 2, no. 3, pp. 192-197, 2008.

[19] X. M. Sun, G. P. Liu, W. Wang, and D. Rees, "L2-gainof systems with input delays and controller temporary failure: zero-order 
hold model," IEEE Transactions on Control Systems Technology, vol. 19, no. 3, pp. 699-706, 2011.

[20] X. Wang, J. Zhao, and Y. Tang, "State tracking model reference adaptive control for switched nonlinear systems with linear uncertain parameters," Journal of Control Theory and Applications, vol. 10, no. 3, pp. 354-358, 2012.

[21] Q. Sang and G. Tao, "Adaptive control of piecewise linear systems: the state tracking case," in Proceedings of the American Control Conference (ACC '10), pp. 4040-4045, July 2010.

[22] C. Wu, C. Li, and J. Zhao, "Switching-based state tracking of model reference adaptive control systems in the presence of intermittent failures of all actuators," International Journal of Adaptive Control and Signal Processing, 2013.

[23] X. P. Xu and G. S. Zhai, "On practical stability and stabilization of hybrid and switched systems," in Proceeding of the 7th International Workshop on Hybrid Systems: Computation and Control (HSCC '04), vol. 2993 of Lecture Notes in Computer Science, pp. 615-630, Berlin, Germany, 2004. 


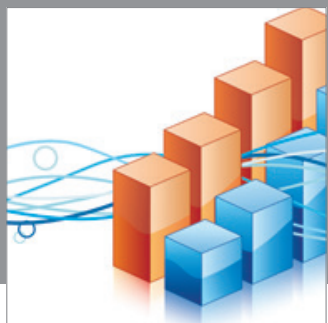

Advances in

Operations Research

mansans

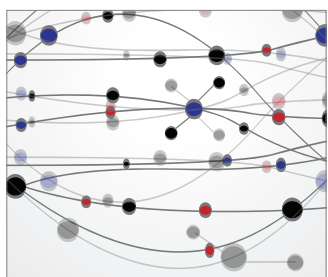

The Scientific World Journal
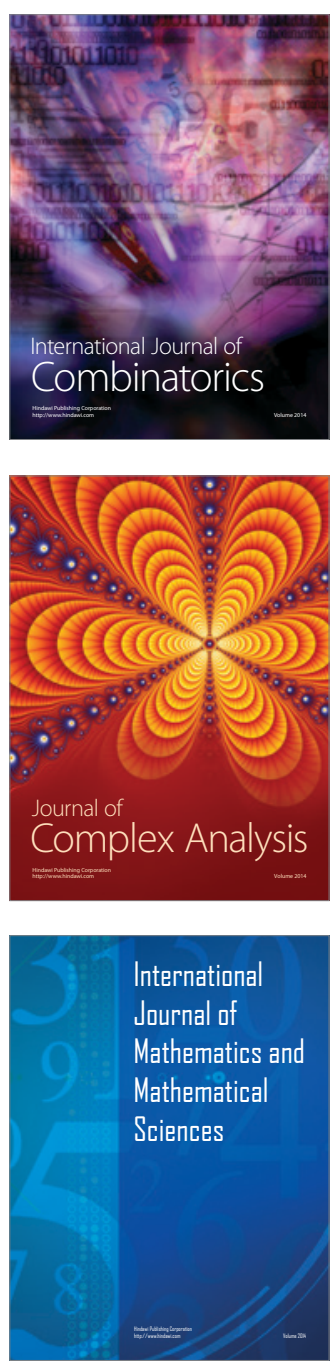
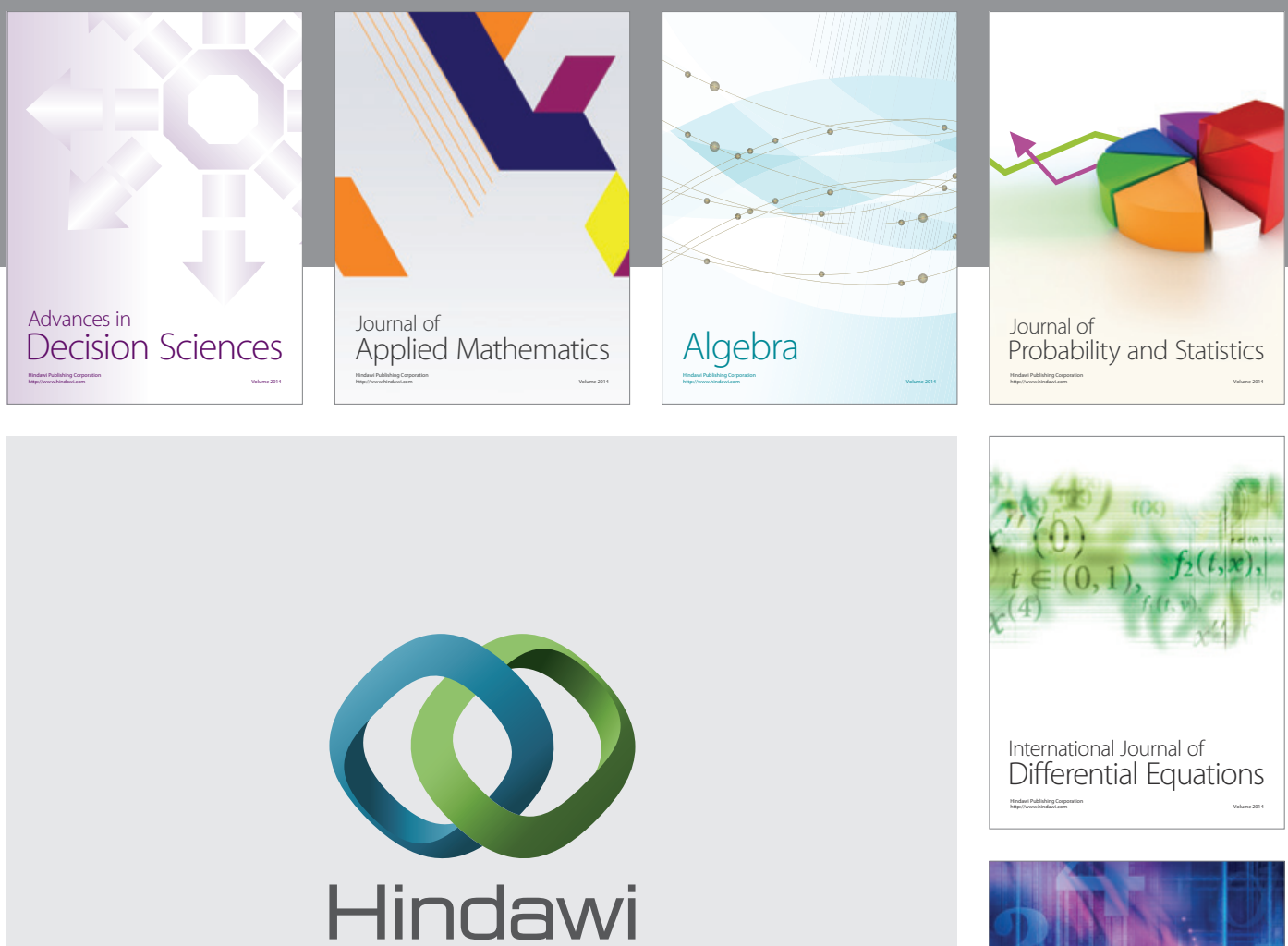

Submit your manuscripts at http://www.hindawi.com
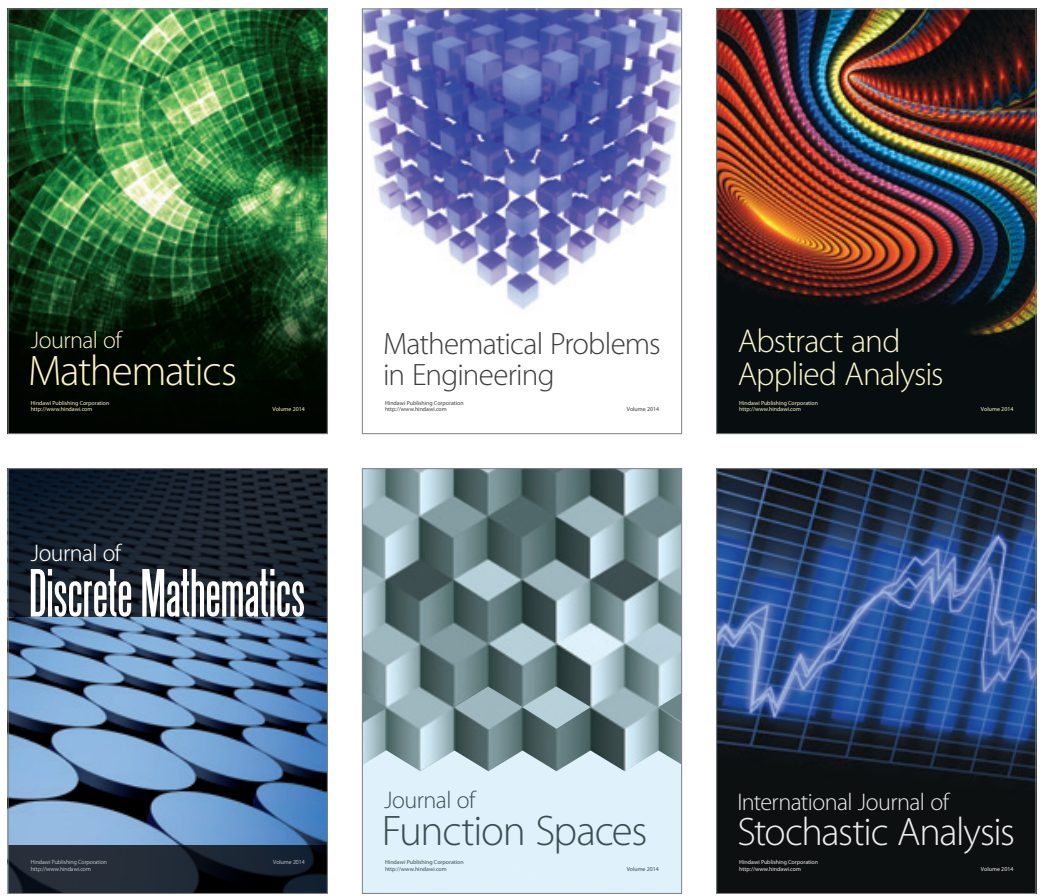

Journal of

Function Spaces

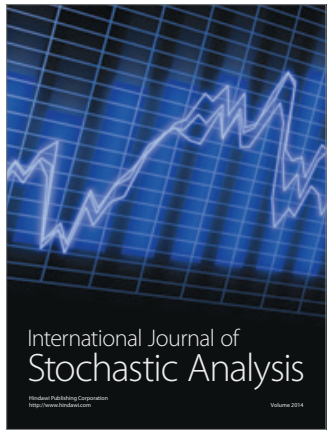

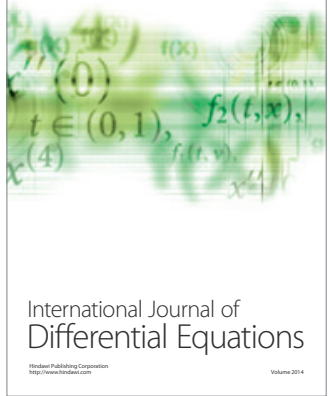
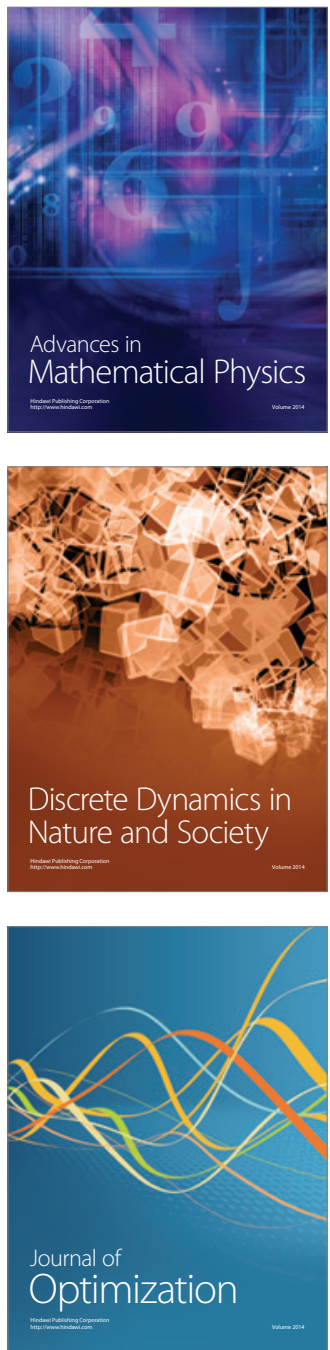